\title{
Ausgezeichnete Rheuma-Forschung
}

Im Rahmen der Jahrestagung der Österreichischen Gesellschaft für Rheumatologie \& Rehabilitation (ÖGR) wurde am 27.11. auch ein Publikationspreis verliehen. Gefördert wird der mit je $€ 1.300$ dotierte Preis der ÖGR vom Biopharma Unternehmen AbbVie.

Jedes Jahr können Mediziner*innen Publikationen aus dem Gebiet der Rheumatologie für den ÖGR- Publikationspreis einreichen. Die Entscheidung über die Vergabe des Preises trifft ein Komitee bestehend aus Präsident, Geschäftsführer und Leiter der medizinisch-wissenschaftlichen Sektion der ÖGR. 2021 ging der Preis an die Mediziner*innen Ass.-Prof. Priv.-Doz. Dr. Johannes Fessler, BSc MSc, Priv.Doz. Mag. Dr. Silvia Hayer, PD und Dr. Daniel Mrak für ihre herausragenden wissenschaftlichen Arbeiten rund um rheumatische Erkrankungen.

\section{Primäres Sjögren-Syndrom}

Das Forschungsteam der MedUni Graz rund um Fessler beschäftigt sich in der Publikation „MH Lymphopenia in Primary Sjögren's Syndrome is Associated with Premature Aging of Naïve CD4+ T Cdxaells" mit dem Einfluss von Alterung, Ernährung und Metabolismus sowie deren Zusammenspiel auf das Immunsystem. Besonderes Augenmerk liegt dabei auf der Regulation autoreaktiver T-Zellen.

T-Zellen von Patient*innen mit Autoimmunerkrankungen leiden unter vorzeitiger Alterung und weisen häufig einen veränderten zellulären Metabolismus auf. Beides kann dabei zu einer erhöhten pro-inflammatorischen Immunantwort und folglich zu chronischer, gewebeschädigender Entzündung führen.

Die Forschergruppe untersucht mögliche Zusammenhänge und die innen zugrundeliegenden Mechanismen von Alterung und Immunstoffwechsel für die Regulation von Immunantworten im Kontext von Autoimmunerkrankungen.

\section{Standardisierte histologische Methoden für Tiermodelle}

Hayer (MedUni Wien) behandelt in ihrer Publikation "ISMASH' Recommendations for Standardised Microscopic Arthritis Scoring of Histological Sections from Inflammatory Arthritis Animal Models" die Standardisierung von Tiermodellen, die bei rheumatoider Arthritis (RA) und Psoriasis-Arthritis (PsA) zur Erforschung pathologischer Mechanismen und Validierung neuer Therapien verwendet werden.

Tiermodelle kommen zwar häufig zum Einsatz, es fehlt aber eine detaillierte Beschreibung und einheitliche Beurteilung der unterschiedlichen histopathologischen Merkmale von Arthritis wie z. B. Gelenkentzündung, Knorpelschäden und Knochenerosionen. Erkenntnisse über die Pathologie aus Maus- und Rattenmodellen sind für zuverlässige Forschungsergebnisse im Bereich der entzündlichen Arthritis von größter Bedeutung. Standardisierte histologische Verarbeitungs- und Bewertungsmethoden mit SMASH-Empfehlungen, die vom BeTheCure-Konsortium formuliert wurden, können zur Vereinheitlichung und Reproduzierbarkeit beitragen.

SARS-CoV-2-Impfung bei Patient*innen, die mit monoklonalen Antikörpern behandelt werden Mrak (Meduni Wien) beschreibt in seiner Studie "SARS-CoV-2 Vaccination in Rituximab-Treated Patients: B Cells Promote Humoral Immune Responses in the Presence of T-Cell-Mediated Immunity", dass Patient*innen, die eine Behandlung mit Rituximab (RTX) erhalten, keine messbaren peripheren B-Zellen, d.h. humorale Immunant- wort nach einer SARS-CoV-2-Impfung, entwickeln.

Es zeigt sich aber, dass Patient*innen, deren periphere B-Zellen sich zumindest teilweise wieder angesiedelt haben, SARS-CoV-2-spezifische Antikörper nach der COVID-19-Impfung bilden. Die SARS-CoV-2-spezifischen T-Zellen, die bei zumindest der Hälfte der Rituximab-Patient*innen nach der Impfung feststellbar sind, scheinen eine protektive Wirkung unabhängig von der humoralen Immunantwort zu haben.

Hinweis des Verlags. Der Verlag bleibt in Hinblick auf geografische Zuordnungen und Gebietsbezeichnungen in veröffentlichten Karten und Institutsadressen neutral.

\section{rheuma plus $2022 \cdot 21: 33$}

https://doi.org/10.1007/s12688-022-

00491-w

(c) The Author(s), under exclusive licence to Springer-Verlag GmbH Austria, ein Teil von Springer Nature 2022

Quelle: Presseaussendung der AbbVie GmbH

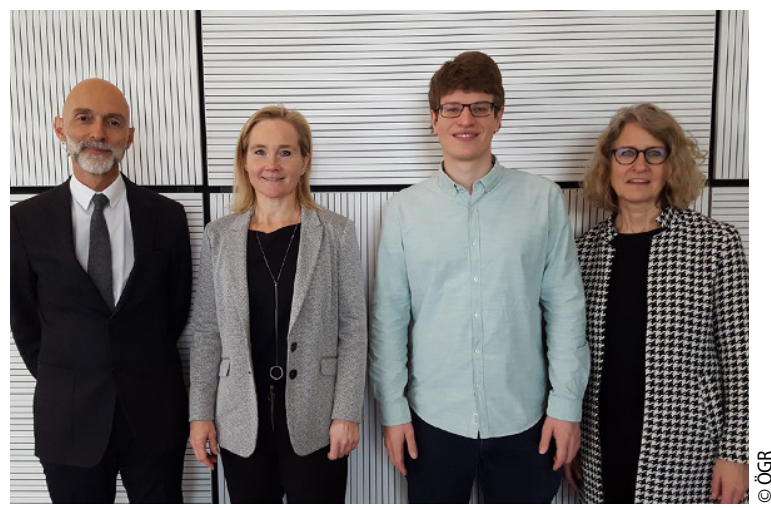

$\Delta$ Die ÖGR Publikationspreisträger*innen Mag. ${ }^{\text {a }}$ Dr. ${ }^{\text {in }}$ Silvia Hayer,

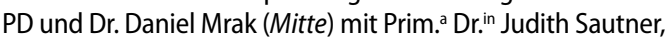
Präsidentin der ÖGR (re.) und Prof. Dr. Clemens Scheinecker, MBA, Leiter der Sektion Wissenschaft (li.) 